AEI-2005-126

hep-th/0507178

June 16, 2018

\title{
Higher-derivative gauge field terms in the M-theory action
}

\author{
Kasper Peeters, Jan Plefka and Steffen Stern \\ Max-Planck-Institut für Gravitationsphysik \\ Albert-Einstein-Institut \\ Am Mühlenberg 1 \\ 14476 Golm, Germany \\ kasper.peeters, jan.plefka, steffen.stern@aei.mpg.de
}

\begin{abstract}
We use superparticle vertex operator correlators in the light-cone gauge to determine the $(D F)^{2} R^{2}$ and $(D F)^{4}$ terms in the M-theory effective action. Our results, when compactified on a circle, reproduce terms in the type-IIA string effective action obtained through string amplitude calculations.
\end{abstract}




\section{Contents}

1 Introduction 2

2 Method 24

2.1 Membrane vertex operator correlators . . . . . . . . . . . . . . . . . . 4

2.2 Evaluation of the Levi-Civita gamma traces . . . . . . . . . . . . . . . 5

2.3 Obtaining the amplitude $\ldots \ldots \ldots \ldots \ldots \ldots \ldots$

\begin{tabular}{llr}
\hline 3 & Results & 8
\end{tabular}

3.1 The amplitudes and the effective action $\ldots \ldots \ldots \ldots \ldots \ldots$

3.2 Cross check via compactification . . . . . . . . . . . . . . . 9

\begin{tabular}{|rr}
\hline 4 Discussion & 10
\end{tabular}

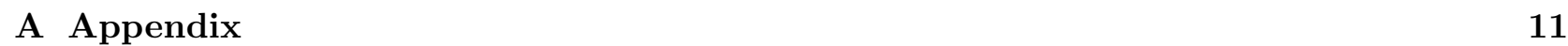

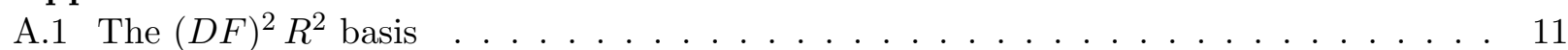

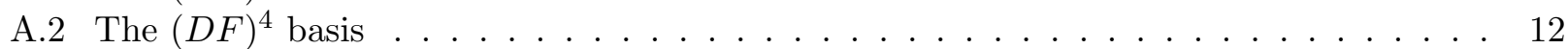

\section{Introduction}

While a substantial effort has been spent on the computation of effective actions for string theory, the situation is not as well developed for M-theory due to our incomplete understanding of its quantum structure. Just as in string theory, the effective action for the massless modes (given by the graviton, the three-form and the gravitino) consists of the lowest-order supergravity action [1] plus an infinite tower of higher-derivative terms. In string theory there exists a variety of methods which can be used to determine this tower of terms. In the background field method one couples the string to a background of supergravity fields. Conformal invariance then demands the vanishing of a set of $\beta$-functionals for these fields, which are interpreted as equations of motion of the effective action. This method is clearly not available in M-theory, as the membrane world-volume action does not satisfy a similar constraint. An alternative method is to employ supersymmetry constraints in order to determine the higher-derivative action. However, determining them in practice along these lines has proved to be hard, both in string theory and in M-theory (see e.g. 2, 3, 4] for a discussion of the present status of this programme).

A third method builds directly upon the S-matrix and extracts the effective action from string scattering amplitudes. This method does admit a certain generalisation to M-theory. From the field theory point of view the higher order corrections are counterterms for the non-renormalisable supergravity theory. Hence one way of determining them is to perform loop computations in $11 \mathrm{~d}$ supergravity, resulting in explicit tensorial expressions of the supergravity fields with cutoff dependent coefficients. These undetermined coefficients may often be fixed by considering a compactification to ten or lower dimensions and comparison to string theory [5, 6]. In order to actually perform these loop calculations it is advisable to make use of a supersymmetric formalism where cancellations are manifest. Although computations based on the covariant on-shell superspace formalism [7, 8] have not yet appeared, an efficient supersymmetric light-cone gauge has been developed by Green, Gutperle and Kwon [9]. In this formalism, one-loop supergravity amplitudes are described by a closed worldline integral of the $11 \mathrm{~d}$ superparticle with vertex operator insertions. The zero-mode structure of the superparticle vertex operators then dictates the vanishing of all one, two and three-point functions at one-loop. The four-point amplitudes are of a very special form, as they factorise into a scalar box 
diagram times the tensorial structure which is completely determined by the fermionic zero mode integral over the vertex operators. For four-gravitons this gives rise to the famous $R^{4}$-term of quartic order in the Riemann tensor.

In the present paper we determine all other superparticle amplitudes for which the tensorial structure is again completely determined by a fermionic zero mode integral. All four-point amplitudes are of this protected nature. The bosonic ones which have not been computed so far are those with four three-form fields or two three-form fields and two gravitons. These lead to terms of the form $(D F)^{4}$ and $(D F)^{2} R^{2}$ in the effective action, where $F=\mathrm{d} C$ with $C$ being the M-theory three-form potential. For the determination of these amplitudes one needs to compute a variety of Levi-Civita traces of $\mathrm{SO}(9)$ Dirac matrices coming from the fermionic zero-mode integral. The resulting tensors generalise the $t_{8}$ tensor for the superstring defined via $\mathrm{SO}(8)$ Dirac matrices. In the present paper we evaluate all these amplitudes and hence determine the structure of the M-theory effective action in this sector. As a test of our results we also check that they reduce to the known string theoretic quartic effective action terms $(D H)^{4}$ and $(D H)^{2} R^{2}$ (with $H=\mathrm{d} B$ ) in 10d computed by Gross and Sloan [10]. Our final expressions may be found in eqs. (3.2) and (3.3).

As a matter of fact the superparticle vertex operator formalism may be lifted to the $11 \mathrm{~d}$ light-cone supermembrane where corresponding vertex operators for the graviton, three-form and gravitino may be defined [11]. These vertices not only reduce to the $11 \mathrm{~d}$ superparticle vertices once one shrinks the membrane space-sheet to a point, but they also reduce to the type-IIA superstring vertices under double-dimensional reduction. It turns out that scattering amplitudes for the supermembrane theory may be defined in this supersymmetric light-cone gauge in analogy with the string and particle descriptions [1, 12]. The tensorial structures of the four-point amplitudes then reduce to precisely the same zero-mode integrals that one encounters in the superparticle computation discussed above. Hence depending on one's personal taste, one may consider our computation of the $(D F)^{4}$ and $(D F)^{2} R^{2}$ terms to arise either from a superparticle or from a supermembrane light-cone formalism.

Let us end this introduction by comparing our method to alternative ways in which higherderivative terms in the M-theory effective action can be computed. Certainly the light-cone gauge has its drawbacks as it is not able to compute all possible amplitudes in a generic background. For example a term of the form $(D F)^{3} R$ will always require the contraction with an $11 \mathrm{~d}$ epsilontensor due to the odd total number of indices. Such terms are hence invisible in light-cone gauge where $R_{+m n p}=0$ and $F_{+m n p}=0$. It would therefore be worth analysing our computations using the covariant formalism for 11d loop-amplitudes as presented recently in [13], which makes use of previous work of Berkovits [14] for the covariant 11d supermembrane.

A completely different method for the computation of quartic terms in the effective action was given by Deser and Seminara in [15, 16, 17]. They argued that one may extract the form of local supergravity counterterms from the nonlocal parts of the tree level four-point amplitudes. Based on this argument they have constructed $(D F)^{4},(D F)^{2} R^{2}$ and $(D F)^{3} R$ counterterms. While our findings agree with theirs for the $(D F)^{2} R^{2}$ terms in the action and we also agree with their nonlocal amplitudes for $(D F)^{4}$, our local $(D F)^{4}$ action differs from the local form obtained in [16].

Finally one might wonder whether Matrix Theory [18] as a contender for the microscopic definition of M-theory can also compute these terms (potentially regularising the divergent prefactors). That is, unfortunately, not the case, as determining these quantum corrections directly from Matrixtheory presumably requires analytical control on the large- $N$ form of the ground state wave function. Investigations of supergraviton scattering amplitudes in Matrix theory have proved to lead to inconsistencies at finite $N$ once one probes beyond the leading supergravity approximation [19]. The leading one-loop Matrix Theory S-Matrix for supergraviton scattering agrees with 11d supergravity, but is in fact completely determined by supersymmetry [20] irrespective of the value of $N$. At twoloops in the $\mathrm{SU}(3)$ model the expected quantum correction of $R^{4}$ is not reproduced in the matrix model as was demonstrated in [19]. 


\section{Method}

\subsection{Membrane vertex operator correlators}

As explained in the introduction, we intend to determine the $(D F)^{2} R^{2}$ and $(D F)^{4}$ terms in the effective action through the computation of superparticle or supermembrane scattering amplitudes. Let us first recall the form of the vertex operators for the physical states which feature in these calculations. Explicit expressions for the membrane vertex operators in the light-cone gauge have been constructed in [1]]. They are given by

$$
\begin{aligned}
V_{h}=h_{a b}\left[D X^{a} D X^{b}-\left\{X^{a}, X^{c}\right\}\left\{X^{b} X^{c}\right\}-i \theta \gamma^{a}\left\{X^{b}, \theta\right\}\right. & \\
& \left.-2 D X^{a} R^{b c} k_{c}-6\left\{X^{a}, X^{c}\right\} R^{b c d} k_{d}+2 R^{a c} R^{b d} k_{c} k_{d}\right] e^{-i k \cdot X}
\end{aligned}
$$

for the graviton and

$$
\begin{aligned}
V_{C}=-C_{a b c} D X^{a}\left\{X^{b}, X^{c}\right\} e^{-i k \cdot X} \\
\quad+F_{a b c d}\left[\left(D X^{a}-\frac{2}{3} R^{a e} k_{e}\right) R^{b c d}-\frac{1}{2}\left\{X^{a}, X^{b}\right\} R^{c d}-\frac{1}{96}\left\{X^{e}, X^{f}\right\} \theta \gamma^{a b c d e f} \theta\right] e^{-i k \cdot X}
\end{aligned}
$$

for the three-form. The $X^{a}\left(\tau, \sigma_{1}, \sigma_{2}\right)$ and $\theta_{\alpha}\left(\tau, \sigma_{1}, \sigma_{2}\right)$ denote the transverse membrane embedding coordinates $(a=1, \ldots, 9$ and $\alpha=1, \ldots, 16)$. Moreover $\{A, B\}:=\epsilon^{r s} \partial_{r} A \partial_{s} B$ where $\partial_{r}:=\partial / \partial \sigma_{r}$ is the Lie bracket of area preserving diffeomorphisms under which the light-cone gauge fixed supermembrane maintains a residual invariance 21]. We also have defined $D X:=\partial_{t} X+\{\omega, X\}$ with $\omega$ denoting the gauge field of the area preserving diffeomorphisms. In the above the symbols $R^{a b c}$ and $R^{a b}$ stand for the fermi bilinears

$$
R^{a b c}=\frac{1}{12} \theta \gamma^{a b c} \theta, \quad R^{a b}=\frac{1}{4} \theta \gamma^{a b} \theta .
$$

Out of these, one can construct $n$-point 1-loop amplitudes via [12, 22]

$$
\mathcal{A}_{1 \text {-loop, } n \text {-point }}=\int \mathrm{d} p^{+} \mathrm{d}^{9} p_{\perp} \operatorname{Tr}\left(\Delta V_{1} \Delta V_{2} \ldots \Delta V_{n}\right) .
$$

where the $V_{n}$ are vertex operators, $\Delta$ a propagator and the trace goes over the Hilbert space. For four-point amplitudes, almost all terms in the expressions (2.1) and (2.2) are, however, irrelevant. This is because a non-zero amplitude requires the saturation of a fermionic $\mathrm{SO}(9)$ integral, according to the identity

$$
\operatorname{Tr}\left(\theta_{\alpha_{1}} \cdots \theta_{\alpha_{N}}\right)_{\theta}=\delta_{N, 16} \epsilon^{\alpha_{1} \ldots \alpha_{16}}
$$

Therefore, in computing four-point correlators, the only relevant terms in the supermembrane vertex operators are those which are already present in the superparticle vertex operators [9]. Those read

$$
\begin{gathered}
V_{h}=2 h_{a b} R^{a c} R^{b d} k_{c} k_{d} e^{-i k \cdot X}, \\
V_{C}=-\frac{2}{3} F_{a b c d} R^{a e} k_{e} R^{b c d} e^{-i k \cdot X} .
\end{gathered}
$$

As in string theory, the amplitudes will contain an overall momentum-dependent factor arising from the correlator of plane-wave exponentials. This factor is expected to exhibit poles corresponding to massless field exchange graphs as well as regular terms corresponding to contact terms. Lacking a firm understanding of these integrals for the supermembrane we will not comment on the momentum dependence any further. Progress on this correlator has been made by passing back to a covariant 
description and working under the assumption that the path integral reduces to a membrane zeromode winding sum while all quantum fluctuations cancel due to supersymmetry [23, 24, 25]. For the case of a three-torus compactification of M-theory this correlator was studied in a three dimensional matrix theory description [26], see also [27] for a related discussion. We will here only assume that the light-cone correlator yields a regular term, so that the amplitudes can be used to determine $(D F)^{2} R^{2}$ and $(D F)^{4}$ terms in the effective action. It should be stressed however, that for a true supermembrane theory reading of our results it remains to be shown that this scalar correlator exists. Alternatively, one could take the point of view that we are computing a superparticle correlator, in which case one has to deal with the loop divergence like in [5].

Explicitly, one now finds that the four-point amplitudes with either gravitons or three-form gauge fields consists of the above-mentioned momentum dependent prefactor times the following tensor structures,

$$
\begin{aligned}
\mathcal{A}_{4 h} & =t_{16}^{a_{1} a_{2} \ldots a_{16}} R_{a_{1} a_{2} a_{3} a_{4}} \cdots R_{a_{13} a_{14} a_{15} a_{16}} \\
\mathcal{A}_{2 h 2 C} & =t_{18}^{a_{1} a_{2} \ldots a_{18}} D_{a_{7}} F_{a_{8} a_{1} a_{2} a_{3}} D_{a_{9}} F_{a_{10} a_{4} a_{5} a_{6}} R_{a_{11} a_{12} a_{13} a_{14}} R_{a_{15} a_{16} a_{17} a_{18}}, \\
\mathcal{A}_{4 C} & =t_{20}^{a_{1} a_{2} \ldots a_{20}} D_{a_{13}} F_{a_{14} a_{1} a_{2} a_{3}} D_{a_{15}} F_{a_{16} a_{4} a_{5} a_{6}} D_{a_{17}} F_{a_{18} a_{7} a_{8} a_{9}} D_{a_{19}} F_{a_{20} a_{10} a_{11} a_{12}} .
\end{aligned}
$$

We have here written Riemann tensors and derivatives of field strengths instead of the linearised expressions in terms of polarisation tensors and momenta, anticipating that these amplitudes are directly responsible for the appearance of contact terms in the effective action. The tensors $t_{16}, t_{18}$ and $t_{20}$ are generalisations of the well-known $t_{8}$ tensor which appears in string theory. Explicitly, these tensors are defined by

$$
\begin{aligned}
& t_{16}^{a_{1} a_{2} \ldots a_{16}}:=\epsilon^{\alpha_{1} \ldots \alpha_{16}} \gamma_{\alpha_{1} \alpha_{2}}^{a_{1} a_{2}} \gamma_{\alpha_{3} \alpha_{4}}^{a_{3} a_{4}} \cdots \gamma_{\alpha_{15} \alpha_{16}}^{a_{15} a_{16}}, \\
& t_{18}^{a_{1} a_{2} \ldots a_{18}}:=\epsilon^{\alpha_{1} \ldots \alpha_{16}} \gamma_{\alpha_{1} \alpha_{2}}^{a_{1} a_{2} a_{3}} \gamma_{\alpha_{3} \alpha_{4}}^{a_{4} a_{5} a_{6}} \gamma_{\alpha_{5} \alpha_{6}}^{a_{7} a_{8}} \ldots \gamma_{\alpha_{15} \alpha_{16}}^{a_{17} a_{18}}, \\
& t_{20}^{a_{1} a_{2} \ldots a_{20}}:=\epsilon^{\alpha_{1} \ldots \alpha_{16}} \gamma_{\alpha_{1} \alpha_{2}}^{a_{1} a_{2} a_{3}} \cdots \gamma_{\alpha_{7} \alpha_{8}}^{a_{10} a_{11} a_{12}} \gamma_{\alpha_{9} \alpha_{10}}^{a_{13} a_{14}} \cdots \gamma_{\alpha_{15} \alpha_{16}}^{a_{19} a_{20}} .
\end{aligned}
$$

Clearly, all information about the structure of the effective action terms is contained in these LeviCivita gamma matrix traces. The $t_{16}$ tensor of course leads to amplitudes which can equivalently be written using the well-known $t_{8} t_{8}$ product. Let us therefore now turn to the computation of the other two traces.

\subsection{Evaluation of the Levi-Civita gamma traces}

Having reduced the problem of computing vertex operator correlators to the evaluation of Levi-Civita gamma matrix traces, one is looking for an effective way to determine the tensor structure of the $t_{18}$ and $t_{20}$ tensors. We here follow a slight modification of the procedure recently described in [28].

The structure of the $t_{16}, t_{18}$ and $t_{20}$ tensors is most conveniently written down in a form contracted with antisymmetric dummy tensors $Y^{a b c}$ and $X^{a b}$, such as to reveal the symmetry of the $t$-tensors. The result has to be a linear combination of all possible contractions of the $X$ and $Y{ }^{1}$ For the $t_{18}$ tensor the decomposition reads

$$
t_{18}^{a_{1} a_{2} \ldots a_{18}} Y_{a_{1} a_{2} a_{3}} Y_{a_{4} a_{5} a_{6}} X_{a_{7} a_{8}} \cdots X_{a_{17} a_{18}}=c_{1} Y^{a b c} Y_{a b c}\left(X^{d e} X_{d e}\right)^{3}+c_{2} Y^{a b c} Y_{a b c} \operatorname{Tr} X^{2} \operatorname{Tr} X^{4}+\ldots
$$

$$
\begin{aligned}
& { }^{1} \text { For completeness, let us mention that in this notation the expression for the contracted } t_{16} \text { tensor equals } \\
& t_{16}^{a_{1} a_{2} \ldots a_{16}} X_{a_{1} a_{2}} \cdots X_{a_{17} a_{18}}= \\
& 105 \cdot 2^{19}\left(-5 \operatorname{Tr}\left(X^{2}\right)^{4}+384 \operatorname{Tr}\left(X^{8}\right)-256 \operatorname{Tr}\left(X^{2}\right) \operatorname{Tr}\left(X^{6}\right)+72 \operatorname{Tr}\left(X^{2}\right)^{2} \operatorname{Tr}\left(X^{4}\right)-48 \operatorname{Tr}\left(X^{4}\right)^{2}\right) .
\end{aligned}
$$


A simple group theory calculation shows that there are 26 possible contractions for the $t_{18}$ tensor: the tensor product

$$
(\boxminus)_{\mathrm{sym}}^{2} \bigotimes(\boxminus)_{\mathrm{sym}}^{6}
$$

contains 26 singlets in $\mathrm{SO}(9)$. In figure 1 these contractions have been visualised by representing $X$ with a black dot with 2 legs, $Y$ with a white dot with 3 legs and a contraction by linking two legs. Repeatedly filling the components of $X$ and $Y$ with random numbers and evaluating both the values of the 26 graphs and the $t_{18}$ contraction (which can be done numerically in a reasonable amount of time), one obtains an overdetermined linear system of equations for the $c_{1}, \ldots, c_{26}$ and thus the tensor structure of $t_{18}$, as shown in table 1 (an alternative method to obtain the $c_{i}$ coefficients, based on a backtracking algorithm, was described in [28]).
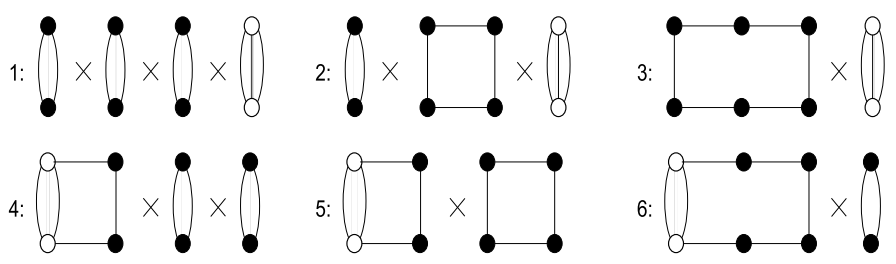

$5:$
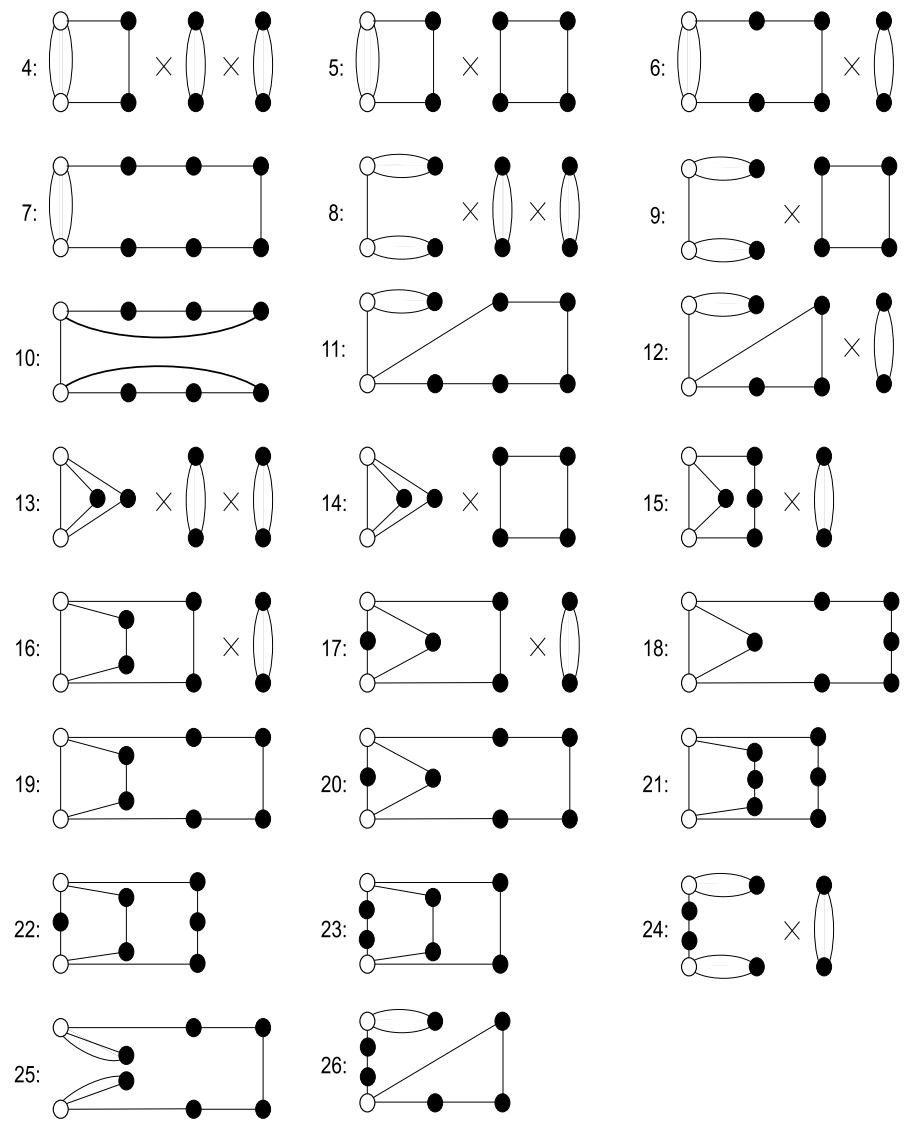

Figure 1: The 26 graphs used to express the gamma trace (2.14), relevant for the $(D F)^{2} R^{2}$ terms in the amplitude. Black and white dots represent $X^{a b}$ and $Y^{a b c}$ tensors respectively.

For the $t_{20}$ one proceeds in a similar way. After contraction with the $X$ and $Y$ tensors we end up with

$$
t_{20}^{a_{1} a_{2} \ldots a_{20}} Y_{a_{1} a_{2} a_{3}} \cdots Y_{a_{10} a_{11} a_{12}} X_{a_{13} a_{14}} \cdots X_{a_{19} a_{20}}=c_{1} Y^{a b c} Y_{a b c} Y^{d e f} Y_{d e f}\left(X^{g h} X_{g h}\right)^{2}+\ldots
$$

In this case there are 83 possible contractions of the $X$ and $Y$ tensors, which follows from the fact 


\begin{tabular}{|c|c|c|}
\hline Scalar $i$ & Tensor structure & factor $c_{i}$ \\
\hline 1 & $\delta^{a_{1} a_{4}} \delta^{a_{2} a_{5}} \delta^{a_{3} a_{6}} \delta^{a_{7} a_{9}} \delta^{a_{8} a_{10}} \delta^{a_{11} a_{13}} \delta^{a_{12} a_{14}} \delta^{a_{15} a_{17}} \delta^{a_{16} a_{18}}$ & 440 \\
\hline 2 & $\delta^{a_{1} a_{4}} \delta^{a_{2} a_{5}} \delta^{a_{3} a_{6}} \delta^{a_{7} a_{9}} \delta^{a_{8} a_{10}} \delta^{a_{11} a_{13}} \delta^{a_{12} a_{15}} \delta^{a_{14} a_{17}} \delta^{a_{16} a_{18}}$ & -2400 \\
\hline 3 & $\delta^{a_{1} a_{4}} \delta^{a_{2} a_{5}} \delta^{a_{3} a_{6}} \delta^{a_{7} a_{9}} \delta^{a_{8} a_{11}} \delta^{a_{10} a_{13}} \delta^{a_{12} a_{15}} \delta^{a_{14} a_{17}} \delta^{a_{16} a_{18}}$ & 2560 \\
\hline 4 & $\delta^{a_{1} a_{4}} \delta^{a_{2} a_{5}} \delta^{a_{3} a_{7}} \delta^{a_{6} a_{9}} \delta^{a_{8} a_{10}} \delta^{a_{11} a_{13}} \delta^{a_{12} a_{14}} \delta^{a_{15} a_{17}} \delta^{a_{16} a_{18}}$ & -7200 \\
\hline 5 & $\delta^{a_{1} a_{4}} \delta^{a_{2} a_{5}} \delta^{a_{3} a_{7}} \delta^{a_{6} a_{9}} \delta^{a_{8} a_{10}} \delta^{a_{11} a_{13}} \delta^{a_{12} a_{15}} \delta^{a_{14} a_{17}} \delta^{a_{16} a_{18}}$ & 17280 \\
\hline 6 & $\delta^{a_{1} a_{4}} \delta^{a_{2} a_{5}} \delta^{a_{3} a_{7}} \delta^{a_{6} a_{9}} \delta^{a_{8} a_{11}} \delta^{a_{10} a_{13}} \delta^{a_{12} a_{14}} \delta^{a_{15} a_{17}} \delta^{a_{16} a_{18}}$ & 23040 \\
\hline 7 & $\delta^{a_{1} a_{4}} \delta^{a_{2} a_{5}} \delta^{a_{3} a_{7}} \delta^{a_{6} a_{9}} \delta^{a_{8} a_{11}} \delta^{a_{10} a_{13}} \delta^{a_{12} a_{15}} \delta^{a_{14} a_{17}} \delta^{a_{16} a_{18}}$ & -46080 \\
\hline 8 & $\delta^{a_{1} a_{4}} \delta^{a_{2} a_{7}} \delta^{a_{3} a_{8}} \delta^{a_{5} a_{9}} \delta^{a_{6} a_{10}} \delta^{a_{11} a_{13}} \delta^{a_{12} a_{14}} \delta^{a_{15} a_{17}} \delta^{a_{16} a_{18}}$ & -720 \\
\hline 9 & $\delta^{a_{1} a_{4}} \delta^{a_{2} a_{7}} \delta^{a_{3} a_{8}} \delta^{a_{5} a_{9}} \delta^{a_{6} a_{10}} \delta^{a_{11} a_{13}} \delta^{a_{12} a_{15}} \delta^{a_{14} a_{17}} \delta^{a_{16} a_{18}}$ & -2880 \\
\hline 10 & $\delta^{a_{1} a_{4}} \delta^{a_{2} a_{7}} \delta^{a_{3} a_{9}} \delta^{a_{5} a_{11}} \delta^{a_{6} a_{13}} \delta^{a_{8} a_{15}} \delta^{a_{10} a_{16}} \delta^{a_{12} a_{17}} \delta^{a_{14} a_{18}}$ & -23040 \\
\hline 11 & $\delta^{a_{1} a_{4}} \delta^{a_{2} a_{7}} \delta^{a_{3} a_{8}} \delta^{a_{5} a_{9}} \delta^{a_{6} a_{11}} \delta^{a_{10} a_{13}} \delta^{a_{12} a_{15}} \delta^{a_{14} a_{17}} \delta^{a_{16} a_{18}}$ & 0 \\
\hline 12 & $\delta^{a_{1} a_{4}} \delta^{a_{2} a_{7}} \delta^{a_{3} a_{8}} \delta^{a_{5} a_{9}} \delta^{a_{6} a_{11}} \delta^{a_{10} a_{13}} \delta^{a_{12} a_{14}} \delta^{a_{15} a_{17}} \delta^{a_{16} a_{18}}$ & 11520 \\
\hline 13 & $\delta^{a_{1} a_{4}} \delta^{a_{2} a_{7}} \delta^{a_{3} a_{9}} \delta^{a_{5} a_{8}} \delta^{a_{6} a_{10}} \delta^{a_{11} a_{13}} \delta^{a_{12} a_{14}} \delta^{a_{15} a_{17}} \delta^{a_{16} a_{18}}$ & 4320 \\
\hline 14 & $\delta^{a_{1} a_{4}} \delta^{a_{2} a_{7}} \delta^{a_{3} a_{9}} \delta^{a_{5} a_{8}} \delta^{a_{6} a_{10}} \delta^{a_{11} a_{13}} \delta^{a_{12} a_{15}} \delta^{a_{14} a_{17}} \delta^{a_{16} a_{18}}$ & -5760 \\
\hline 15 & $\delta^{a_{1} a_{4}} \delta^{a_{2} a_{7}} \delta^{a_{3} a_{9}} \delta^{a_{5} a_{8}} \delta^{a_{6} a_{11}} \delta^{a_{10} a_{13}} \delta^{a_{12} a_{14}} \delta^{a_{15} a_{17}} \delta^{a_{16} a_{18}}$ & -46080 \\
\hline 16 & $\delta^{a_{1} a_{4}} \delta^{a_{2} a_{7}} \delta^{a_{3} a_{9}} \delta^{a_{5} a_{11}} \delta^{a_{6} a_{13}} \delta^{a_{8} a_{12}} \delta^{a_{10} a_{14}} \delta^{a_{15} a_{17}} \delta^{a_{16} a_{18}}$ & 23040 \\
\hline 17 & $\delta^{a_{1} a_{7}} \delta^{a_{2} a_{9}} \delta^{a_{3} a_{11}} \delta^{a_{4} a_{8}} \delta^{a_{5} a_{10}} \delta^{a_{6} a_{13}} \delta^{a_{12} a_{14}} \delta^{a_{15} a_{17}} \delta^{a_{16} a_{18}}$ & -11520 \\
\hline 18 & $\delta^{a_{1} a_{4}} \delta^{a_{2} a_{7}} \delta^{a_{3} a_{9}} \delta^{a_{5} a_{8}} \delta^{a_{6} a_{11}} \delta^{a_{10} a_{13}} \delta^{a_{12} a_{15}} \delta^{a_{14} a_{17}} \delta^{a_{16} a_{18}}$ & 92160 \\
\hline 19 & $\delta^{a_{1} a_{4}} \delta^{a_{2} a_{7}} \delta^{a_{3} a_{9}} \delta^{a_{5} a_{11}} \delta^{a_{6} a_{13}} \delta^{a_{8} a_{12}} \delta^{a_{10} a_{15}} \delta^{a_{14} a_{17}} \delta^{a_{16} a_{18}}$ & -92160 \\
\hline 20 & $\delta^{a_{1} a_{7}} \delta^{a_{2} a_{9}} \delta^{a_{3} a_{11}} \delta^{a_{4} a_{8}} \delta^{a_{5} a_{10}} \delta^{a_{6} a_{13}} \delta^{a_{12} a_{15}} \delta^{a_{14} a_{17}} \delta^{a_{16} a_{18}}$ & 0 \\
\hline 21 & $\delta^{a_{1} a_{4}} \delta^{a_{2} a_{7}} \delta^{a_{3} a_{9}} \delta^{a_{5} a_{11}} \delta^{a_{6} a_{13}} \delta^{a_{8} a_{15}} \delta^{a_{10} a_{17}} \delta^{a_{12} a_{16}} \delta^{a_{14} a_{18}}$ & 46080 \\
\hline 22 & $\delta^{a_{1} a_{7}} \delta^{a_{2} a_{9}} \delta^{a_{3} a_{11}} \delta^{a_{4} a_{8}} \delta^{a_{5} a_{13}} \delta^{a_{6} a_{15}} \delta^{a_{10} a_{14}} \delta^{a_{12} a_{17}} \delta^{a_{16} a_{18}}$ & 92160 \\
\hline 23 & $\delta^{a_{1} a_{7}} \delta^{a_{2} a_{9}} \delta^{a_{3} a_{11}} \delta^{a_{4} a_{13}} \delta^{a_{5} a_{15}} \delta^{a_{6} a_{17}} \delta^{a_{8} a_{14}} \delta^{a_{10} a_{16}} \delta^{a_{12} a_{18}}$ & 0 \\
\hline 24 & $\delta^{a_{1} a_{7}} \delta^{a_{2} a_{8}} \delta^{a_{3} a_{9}} \delta^{a_{4} a_{11}} \delta^{a_{5} a_{12}} \delta^{a_{6} a_{13}} \delta^{a_{10} a_{14}} \delta^{a_{15} a_{17}} \delta^{a_{16} a_{18}}$ & 5760 \\
\hline 25 & $\delta^{a_{1} a_{7}} \delta^{a_{2} a_{8}} \delta^{a_{3} a_{9}} \delta^{a_{4} a_{11}} \delta^{a_{5} a_{12}} \delta^{a_{6} a_{13}} \delta^{a_{10} a_{15}} \delta^{a_{14} a_{17}} \delta^{a_{16} a_{18}}$ & 0 \\
\hline 26 & $\delta^{a_{1} a_{7}} \delta^{a_{2} a_{8}} \delta^{a_{3} a_{9}} \delta^{a_{4} a_{11}} \delta^{a_{5} a_{13}} \delta^{a_{6} a_{15}} \delta^{a_{10} a_{12}} \delta^{a_{14} a_{17}} \delta^{a_{16} a_{18}}$ & -46080 \\
\hline
\end{tabular}

Table 1: Decomposition of the $t_{18}$ tensor, defined in (2.11), in terms of Lorentz singlets.

that the tensor product

$$
(\boxminus)_{\mathrm{sym}}^{4} \bigotimes(\boxminus)_{\mathrm{sym}}^{4}
$$

contains 83 singlets in $\mathrm{SO}(9)$. Because the procedure that leads to the determination of the 83 Lorentz singlets and their coefficients is entirely the same as for the $t_{18}$ tensor, we will refrain from spelling out these results.

There is, however, one subtle point which is worth mentioning. We are computing the membrane vertex operator correlators in the light-cone gauge, i.e. the indices take values in $\mathrm{SO}(9)$. However, when the tensor product (2.17) is computed in $\mathrm{SO}(11)$, one finds one additional singlet. Therefore, when the 84 basis elements in $\mathrm{SO}(11)$ are reduced to $\mathrm{SO}(9)$, one has to find one non-trivial identity. When, subsequently, the tensors $X$ and $Y$ are replaced with $D F$ tensors (see the next subsection), this identity leads to one non-trivial identity between the various elements in the basis of $(D F)^{4}$ invariants. This identity is spelled out in (A.6). It implies that the light-cone computation will always leave one linear combination of terms in the covariant action undetermined. However, as we will see below, a covariant computation of the four-point amplitude resulting from this linear combination yields a vanishing result. While the ambiguity thus restricts our ability to determine the full effective action, it is irrelevant for the covariantisation of our light-cone amplitudes. 


\subsection{Obtaining the amplitude}

The final step towards the amplitude is replacing $X X \rightarrow R, X Y \rightarrow D F$ and adequately symmetrising, e.g. for the $(D F)^{2} R^{2}$ amplitude

$$
\begin{aligned}
Y_{a_{1} a_{2} a_{3}} Y_{a_{4} a_{5} a_{6}} X_{a_{7} a_{8}} X_{a_{9} a_{10}} & X_{a_{11} a_{12}} X_{a_{13} a_{14}} X_{a_{15} a_{16}} X_{a_{17} a_{18}} \\
& \rightarrow\left(D_{\left[a_{7}\right.} F_{\left.a_{8}\right] a_{1} a_{2} a_{3}} D_{\left[a_{9}\right.} F_{\left.a_{10}\right] a_{4} a_{5} a_{6}} R_{a_{11} a_{12} a_{13} a_{14}} R_{a_{15} a_{16} a_{17} a_{18}}\right)_{\mathrm{sym}} .
\end{aligned}
$$

Here the suffix "sym" denotes symmetrisation in the 6 index pairs $\left[a_{7} a_{8}\right], \ldots,\left[a_{17} a_{18}\right]$ and the two index triplets $\left[a_{1} a_{2} a_{3}\right],\left[a_{4} a_{5} a_{6}\right]$. The amplitude is then simply

$$
\begin{aligned}
& \mathcal{A}_{(D F)^{2} R^{2}}=\left(c_{1} \delta^{a_{1} a_{4}} \delta^{a_{2} a_{5}} \delta^{a_{3} a_{6}} \delta^{a_{7} a_{9}} \delta^{a_{8} a_{10}} \delta^{a_{11} a_{13}} \delta^{a_{12} a_{14}} \delta^{a_{15} a_{17}} \delta^{a_{16} a_{18}}+\ldots\right) \\
& \times\left(D_{\left[a_{7}\right.} F_{\left.a_{8}\right] a_{1} a_{2} a_{3}} D_{\left[a_{9}\right.} F_{\left.a_{10}\right] a_{4} a_{5} a_{6}} R_{a_{11} a_{12} a_{13} a_{14}} R_{a_{15} a_{16} a_{17} a_{18}}\right)_{\mathrm{sym}} .
\end{aligned}
$$

Eventually, one wants to bring the amplitude in an appealing form, writing it in terms of a (suitably chosen) basis. To do so, one has to use, besides the simple monoterm symmetries, multiterm symmetries, which can be tackled with the method of Young projectors [28]. A nice consequence of our method of calculation is that it leads straightaway to a covariant form of the action, by virtue of the fact that the vertex operators are written in terms of linearised Riemann tensors and linearised derivatives of field strengths.

\section{Results}

\subsection{The amplitudes and the effective action}

Using the method outlined in the previous section, the two-graviton/two-threeform and four-threeform amplitudes can now be computed. Summarising, the four-particle amplitudes are then given by the following expressions (in terms of the Fulling basis [29, 2] and the tensor monomial basis given in the appendix):

$$
\begin{aligned}
& \mathcal{A}_{R^{4}}=2^{20}\left(-192 A_{4}+768 A_{7}\right), \\
& \begin{aligned}
& \mathcal{A}_{(D F)^{2} R^{2}=2^{21} \cdot 3^{3}\left(-24 B_{5}-48 B_{8}-24 B_{10}-6 B_{12}-\right.} 12 B_{13}+12 B_{14} \\
&\left.+8 B_{16}-4 B_{20}+B_{22}+4 B_{23}+B_{24}\right), \\
& \mathcal{A}_{(D F)^{4}}=2^{19} \cdot 3^{7}\left(3 C_{5}+C_{6}-9 C_{8}+C_{9}-72 C_{12}+9 C_{14}+18 C_{17}-9 C_{18}-72 C_{19}-C_{22}\right) .
\end{aligned}
\end{aligned}
$$

We should emphasise that these expressions are amplitudes, even though we have expressed them in terms of the effective action contact terms which can produce them. As explained in the appendix, there are 6 linear combinations of $(D F)^{2} R^{2}$ terms and 9 linear combinations of $(D F)^{4}$ terms in the effective action which lead to a vanishing amplitude. Their coefficients are therefore not determined by the expressions above. This is similar to the well-known fact that the term

$$
\int \mathrm{d}^{10} x \epsilon_{m n r_{1} \ldots r_{8}} \epsilon_{m n s_{1} \ldots s_{8}} R_{r_{1} r_{2} s_{1} s_{2}} \cdots R_{r_{7} r_{8} s_{7} s_{8}}
$$

in the string effective action leads to a vanishing four-graviton amplitude. In order to determine its

coefficient, a computation of the five-graviton amplitude is required. 
The covariant effective action for the $(D F)^{2} R^{2}$ and the $(D F)^{4}$ terms is thus given by (3.2) and (3.3) (with the $B_{i}$ and $C_{i}$ now interpreted as covariant terms in the action), plus an undetermined linear sum of the $Z_{i}$ and $\tilde{Z}_{i}$ combinations given in (A.3) and A.7. $^{2}$. Unfortunately the number of undetermined coefficients in the action is thus rather large (6 and 9 coefficients respectively) and determining these requires information from higher-point amplitudes. We will not attempt that here (note, however, that a similar ambiguity is also present in the superstring effective action, where e.g. the $(D H)^{2} R^{2}$ terms are determined by a four-point calculation only up to a four-parameter family of terms 30,31$]$ ).

Finally, we have also verified that when our one-loop amplitudes are divided by the product of the Mandelstam variables stu, they become identical to the tree-level exchange amplitudes with the same external particles. This lends support to the method followed by Deser \& Seminara for the construction of higher-derivative counterterms [15, 16, 17]. While we agree on the form of the $(D F)^{2} R^{2}$ effective action, the local $(D F)^{4}$ action obtained in [16] yields an amplitude which differs from the one obtained from our expression (3.3). We do, however, agree with [16] on the original, nonlocal amplitude.

\subsection{Cross check via compactification}

Upon compactification of our eleven-dimensional results to ten dimensions, it should be possible to match our amplitudes with known expressions for the two-graviton/two-twoform and four-twoform amplitudes in type-IIA string theory. These were computed a long time ago [10]. Writing the elevendimensional indices as $A=(a, 11)$, the compactification rule is

$$
F_{11 a b c}=H_{a b c}
$$

The string computations of 10], which were done in the Ramond-Neveu-Schwarz formalism, show that the $(D H)^{2} R^{2}$ and $(D H)^{4}$ terms in the effective action are obtained from the $R^{4}$ action by shifting the spin connection with the curvature of the two-form gauge field (the torsion),

$$
\omega \rightarrow \omega+H_{(3)} \quad \rightarrow \quad R_{a b c d} \rightarrow R_{a b c d}+D_{[a} H_{b] c d} .
$$

The amplitudes are thus simply obtained by replacing an appropriate number of Riemann tensors with derivatives of the gauge field curvature in the expression

$$
\mathcal{A}_{R^{4}}=t_{8}^{a_{1} \cdots a_{8}} t_{8}^{b_{1} \cdots b_{8}} R_{a_{1} a_{2} b_{1} b_{2}} R_{a_{3} a_{4} b_{3} b_{4}} R_{a_{5} a_{6} b_{5} b_{6}} R_{a_{7} a_{8} b_{7} b_{8}} .
$$

Note that due to the fact that $D_{[a} H_{b] c d}$ has a different symmetry structure compared to $R_{a b c d}$,

$$
R_{a b c d}=R_{c d a b} \quad \text { vs. } \quad D_{[a} H_{b] c d}=-D_{[c} H_{d] d a b}
$$

one must not first perform the contractions in (3.7) (see e.g. 2] for the result) and then perform the substitution (3.6). Instead, the substitution must be made at the level of (3.7) directly.

As it should be, we now find that when we compactify our $(D F)^{2} R^{2}$ and $(D F)^{4}$ amplitudes, they precisely match the corresponding $(D H)^{2} R^{2}$ resp. $(D H)^{4}$ amplitudes of [10]. This match of the compactified action provides a strong consistency check on our computations.

\footnotetext{
${ }^{2}$ The $\tilde{Z}_{i}$ terms also contain the $\mathrm{SO}(9)$ identity discussed at the end of section 2.2 so that no further ambiguity is introduced by this identity.
} 


\section{Discussion}

We have computed the $(D F)^{2} R^{2}$ and $(D F)^{4}$ terms in the M-theory effective action using a light-cone gauge superparticle or supermembrane calculation. Let us conclude by discussing some open issues and possible applications of our results.

In order to compute terms in the effective action which do not contain derivatives on the gauge fields, like $R^{3} F^{2}$, it is necessary to analyse higher-point amplitudes. In this case, it becomes possible to saturate the fermionic zero modes without restricting to the superparticle terms (2.6) in the supermembrane vertex operators. At first sight, this seems to imply that one needs full control over the bosonic correlators, which at least for the time being is not sufficiently understood for the supermembrane. However, it may be that certain simplifications occur because of the fact that e.g. the $(D F)^{2} R^{2}$ and $F^{2} R^{3}$ terms in the effective action are related to each other by nonlinear supersymmetry. A similar supersymmetry relation is, presumably, responsible for the fact that the insertion point integrals of string five-point amplitudes reduce to extremely simple expressions [30].

One of our motivations for the computation presented here is given by recent results of Damour and Nicolai [32]. Their work is concerned with the study of dynamics near space-like singularities in eleven dimensions, following the seminal work of Belinsky, Khalatnikov and Lifshitz [33, 34]. In the late-time limit, it has been known for some time [35] that supergravity reduces to a point particle sigma model on the infinite dimensional coset space $E_{10} / K\left(E_{10}\right)$. It was shown in [32] that the higher-derivative $R^{4}$ terms in the M-theory effective action are also encoded, in an intriguing way, in the structure of the $E_{10}$ root space. It would be very interesting to analyse whether the $(D F)^{2} R^{2}$ and $(D F)^{4}$ terms agree in a similar way with the structure expected from $E_{10}$. Such a match would provide strong support for an entirely new perspective on the construction of the M-theory effective action.

\section{Acknowledgements}

We thank Stanley Deser for comments on a draft of this paper and for correspondence concerning the results of [16]. 


\section{A Appendix}

\section{A.1 The $(D F)^{2} R^{2}$ basis}

If we impose the linearised lowest-order equations of motion ${ }^{3}$, there are 24 possible $(D F)^{2} R^{2}$ terms in the action, which follows from the fact that the tensor product

$$
(\boxminus)_{\mathrm{sym}}^{2} \bigotimes(\boxminus)_{\mathrm{sym}}^{2}
$$

contains 24 singlets in $\mathrm{SO}(11)$. We choose a basis given by

$$
\begin{aligned}
B_{1} & =R_{a b c d} R_{e f g h} D^{e} F^{a g h}{ }_{i} D^{c} F^{b d f i} & B_{13} & =R_{a b c d} R_{e}{ }^{a} f^{c} D_{i} F^{b f}{ }_{g h} D^{i} F^{d e g h} \\
B_{2} & =R_{a b c d} R_{e f g h} D^{e} F^{a c g}{ }_{i} D^{h} F^{b d f i} & B_{14} & =R_{a b c d} R_{e}{ }^{a}{ }_{f}{ }^{c} D_{i} F^{b d}{ }_{g h} D^{i} F^{e f g h} \\
B_{3} & =R_{a b c d} R_{e f g h} D^{e} F^{a c g}{ }_{i} D^{f} F^{b d h i} & B_{15} & =R_{a b c d} R_{e}{ }^{c}{ }_{f}{ }^{b} D^{b} F^{f}{ }_{g h i} D^{e} F^{d g h i} \\
B_{4} & =R_{a b c d} R_{e f g h} D_{i} F^{c d g h} D^{f} F^{i a b e} & B_{16} & =R_{a b c d} R_{e}{ }^{a} f^{c} D^{b} F^{d}{ }_{g h i} D^{e} F^{f g h i} \\
B_{5} & =R_{a b c d} R_{e f g}{ }^{d} D^{a} F^{b c}{ }_{h i} D^{e} F^{f g h i} & B_{17} & =R_{a b c d} R_{e}{ }^{a} f^{c} D^{b} F^{e}{ }_{g h i} D^{d} F^{f g h i} \\
B_{6} & =R_{a b c d} R_{e f g}{ }^{d} D^{a} F^{b e}{ }_{h i} D^{c} F^{f g h i} & B_{18} & =R_{a b c d} R_{e}{ }^{a}{ }_{f}{ }^{c} D_{i} F^{e f}{ }_{g h} D^{d} F^{b g h i} \\
B_{7} & =R_{a b c d} R_{e f g}{ }^{d} D^{a} F^{b e}{ }_{h i} D^{g} F^{c f h i} & B_{19} & =R_{a b c d} R_{e f}{ }^{c d} D_{i} F^{a e}{ }_{g h} D^{i} F^{b g h} \\
B_{8} & =R_{a b c d} R_{e f g}{ }^{d} D^{a} F^{c e}{ }_{h i} D^{b} F^{f g h i} & B_{20} & =R_{a b c d} R_{e f}{ }^{c d} D^{a} F^{e}{ }_{g h i} D^{b} F^{f g h i} \\
B_{9} & =R_{a b c d} R_{e f g}{ }^{d} D^{a} F^{c e}{ }_{h i} D^{f} F^{b g h i} & B_{21} & =R_{a b c d} R_{e f}{ }^{c d} D^{a} F^{e}{ }_{g h i} D^{f} F^{b g h i} \\
B_{10} & =R_{a b c d} R_{e f g}{ }^{d} D_{i} F^{c e g h} D^{i} F^{a b f h} & B_{22} & =R_{a b c d} R_{e}{ }^{a c d} D^{b} F_{f g h i} D^{e} F^{f g h i} \\
B_{11} & =R_{a b c d} R_{e f g}{ }^{d} D_{h} F^{a b f}{ }_{i} D^{i} F^{c e g h} & B_{23} & =R_{a b c d} R_{e}{ }^{a c d} D_{i} F^{b}{ }_{f g h} D^{i} F^{e f g h} \\
B_{12} & =R_{a b c d} R_{e f g}{ }^{d} D^{c} F^{e f}{ }_{h i} D^{g} F^{b a h i} & B_{24} & =R_{a b c d} R^{a b c d} D_{e} F_{f g h i} D^{f} F^{e g h i}
\end{aligned}
$$

The four-point amplitudes resulting from these contact terms are, however, not all independent. We find that the following 6 linear combinations lead to a vanishing four-point amplitude:

$$
\begin{aligned}
Z_{1}= & 48 B_{1}+48 B_{2}-48 B_{3}+36 B_{4}+96 B_{6}+48 B_{7}-48 B_{8}+96 B_{10} \\
& \quad+12 B_{12}+24 B_{13}-12 B_{14}+8 B_{15}+8 B_{16}-16 B_{17}+6 B_{19}+2 B_{22}+B_{24}, \\
Z_{2}=- & 48 B_{1}-48 B_{2}-24 B_{4}-24 B_{5}+48 B_{6}-48 B_{8}-24 B_{9}-72 B_{10} \\
\quad & \quad-24 B_{13}+24 B_{14}-B_{22}+4 B_{23}, \\
Z_{3}= & 12 B_{1}+12 B_{2}-24 B_{3}+9 B_{4}+48 B_{6}+24 B_{7}-24 B_{8}+24 B_{10}+6 B_{12}+6 B_{13} \\
& \quad+4 B_{15}-4 B_{17}+3 B_{19}+2 B_{21}, \\
Z_{4}= & 12 B_{1}+12 B_{2}-12 B_{3}+9 B_{4}+24 B_{6}+12 B_{7}-12 B_{8}+24 B_{10}+3 B_{12} \\
\quad & \quad+6 B_{13}+4 B_{15}-4 B_{17}+2 B_{20}, \\
Z_{5}= & 4 B_{3}-8 B_{6}-4 B_{7}+4 B_{8}-B_{12}-2 B_{14}+4 B_{18}, \\
Z_{6}= & B_{4}+2 B_{11} .
\end{aligned}
$$

In order to determine the coefficients of these linear combinations of terms in the effective action it is necessary to consider amplitudes with more external legs (which we will not attempt in the present paper).

\footnotetext{
${ }^{3}$ This we can do, as field redefinitions of $g_{a b}$ and $C_{a b c}$ allow us to always remove terms proportional to the equations of motion at the first level of higher derivative corrections.
} 


\section{A.2 The $(D F)^{4}$ basis}

Just like the basis of $(D F)^{2} R^{2}$ terms, the basis for the $(D F)^{4}$ terms consists, at least at linearised on-shell level, of 24 elements: the tensor product

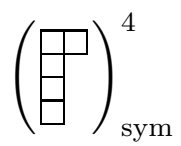

contains 24 singlets in $\mathrm{SO}(11)$. We choose these to be

$$
\begin{aligned}
& C_{1}=D_{a} F_{b c d e} D^{a} F^{b c d e} D_{f} F_{g h i j} D^{f} F^{g h i j} \quad C_{13}=D_{a} F_{b c d e} D^{a} F^{b}{ }_{f g h} D^{c} F^{f g}{ }_{i j} D^{d} F^{e h i j} \\
& C_{2}=D_{a} F_{b c d e} D^{a} F_{f}^{b c d} D^{e} F_{g h i j} D^{f} F^{g h i j} \quad C_{14}=D_{a} F_{b c d e} D^{a} F_{f g h}^{b} D_{i} F^{c d f}{ }_{j} D^{i} F^{e g h j} \\
& C_{3}=D_{a} F_{b c d e} D^{a} F^{b c d}{ }_{f} D_{g} F^{e}{ }_{h i j} D^{g} F^{f h i j} \quad C_{15}=D_{a} F_{b c d e} D^{a} F_{f g h i} D^{b} F^{c d f}{ }_{j} D^{g} F^{e h i j} \\
& C_{4}=D_{a} F_{b c d e} D^{a} F^{b c}{ }_{f g} D^{d} F^{e}{ }_{h i j} D^{f} F^{g h i j} \quad C_{16}=D_{a} F_{b c d e} D^{a} F_{f g h i} D^{b} F^{f g h}{ }_{j} D^{i} F^{c d e j} \\
& C_{5}=D_{a} F_{b c d e} D^{a} F_{f g h}^{b} D_{i} F^{c d e}{ }_{j} D^{f} F^{g h i j} \quad C_{17}=D_{a} F_{b c d e} D^{b} F^{a c}{ }_{f g} D_{h} F^{d f}{ }_{i j} D^{i} F^{e g h j} \\
& C_{6}=D_{a} F_{b c d e} D^{a} F_{f g h}^{b} D_{i} F^{c d e}{ }_{j} D^{j} F^{f g h i} \quad C_{18}=D_{a} F_{b c d e} D^{b} F^{a}{ }_{f g h} D_{i} F^{c d f}{ }_{j} D^{j} F^{e g h i} \\
& C_{7}=D_{a} F_{b c d e} D^{b} F^{a c}{ }_{f g} D^{d} F^{e}{ }_{h i j} D^{h} F^{f g i j} \quad C_{19}=D_{a} F_{b c d e} D^{b} F^{c}{ }_{f g h} D^{f} F^{d g}{ }_{i j} D^{i} F^{a e h j} \\
& C_{8}=D_{a} F_{b c d e} D^{b} F^{a c}{ }_{f g} D_{h} F^{d e}{ }_{i j} D^{i} F^{f g h j} \quad C_{20}=D_{a} F_{b c d e} D^{b} F_{f g h}^{c} D^{f} F^{a d}{ }_{i j} D^{i} F^{e g h j} \\
& C_{9}=D_{a} F_{b c d e} D^{b} F_{f g h i} D^{f} F_{j h i}^{g h} D^{j} F^{a c d e} \quad C_{21}=D_{a} F_{b c d e} D^{b} F_{f g h}^{c} D^{d} F^{f g}{ }_{i j} D^{h} F^{a e i j} \\
& C_{10}=D_{a} F_{b c d e} D^{a} F^{b c}{ }_{f g} D_{h} F^{d f}{ }_{i j} D^{i} F^{e g h j} \quad C_{22}=D_{a} F_{b c d e} D^{b} F_{f g h i} D^{f} F^{c d e}{ }_{j} D^{j} F^{a g h i} \\
& C_{11}=D_{a} F_{b c d e} D^{a} F_{f g}^{b c} D^{d} F^{f}{ }_{h i j} D^{g} F^{e h i j} \quad C_{23}=D_{a} F_{b c d e} D^{b} F_{f g h i} D^{f} F^{c d g}{ }_{j} D^{j} F^{a e h i} \\
& C_{12}=D_{a} F_{b c d e} D^{a} F^{b}{ }_{f g h} D^{c} F^{d f}{ }_{i j} D^{i} F^{e g h j} \quad C_{24}=D_{a} F_{b c d e} D^{b} F_{f g h i} D^{f} F^{a c d}{ }_{j} D^{g} F^{e h i j} \text {. }
\end{aligned}
$$

However, when the indices are restricted to the transversal $\mathrm{SO}(9)$ sector, the tensor product (A.4) only contains 23 singlets. The $\mathrm{SO}(9)$ identity which relates the 24 th basis element to the others can be found by using the fact that $\epsilon_{10}$ is always zero in $\mathrm{SO}(9)$. This implies that

$$
\begin{aligned}
0= & \epsilon^{a_{1} \cdots a_{10}} \epsilon^{b_{1} \cdots b_{10}} D_{a_{1}} F_{b_{1} b_{2} b_{3} b_{4}} D_{a_{2}} F_{a_{3} b_{5} b_{6} b_{7}} D_{a_{4}} F_{a_{5} a_{6} b_{8} b_{9}} D_{a_{7}} F_{a_{8} a_{9} a_{10} b_{10}} \\
= & \mathrm{const} \cdot\left(-C_{1}+8 C_{2}+16 C_{3}-96 C_{4}-32 C_{6}+144 C_{8}-16 C_{9}+96 C_{11}+1728 C_{12}-288 C_{13}\right. \\
& \left.-144 C_{14}-32 C_{16}-576 C_{17}+288 C_{18}+1728 C_{19}-144 C_{21}+144 C_{23}\right)+ \text { off shell terms . }
\end{aligned}
$$

The four-point amplitudes which are generated by the $C_{i}$ terms are again not all independent. It turns out that 9 linear combinations of basis elements lead to a vanishing amplitude. These combinations are given by

$$
\begin{aligned}
& \tilde{Z}_{1}=-C_{3}+12 C_{4}-6 C_{5}+72 C_{7}-9 C_{8}-C_{9}+54 C_{10}-6 C_{11}-144 C_{12}+18 C_{14}-27 C_{18}+18 C_{21} \\
& \tilde{Z}_{2}=C_{3}-6 C_{5}-18 C_{7}+9 C_{8}+C_{9}+6 C_{11}+9 C_{18}+18 C_{23}, \\
& \tilde{Z}_{3}=C_{1}+96 C_{4}-96 C_{5}+32 C_{6}+288 C_{7}+64 C_{9}+32 C_{22} \\
& \tilde{Z}_{4}=-C_{10}+2 C_{12}+2 C_{20} \\
& \tilde{Z}_{5}=C_{7}+C_{10}+4 C_{19} \\
& \tilde{Z}_{6}=-C_{7}-C_{10}+2 C_{17} \\
& \tilde{Z}_{7}=C_{1}-8 C_{2}+32 C_{6}+32 C_{9}+32 C_{16} \\
& \tilde{Z}_{8}=-C_{2}-12 C_{4}+12 C_{5}-4 C_{9}-12 C_{11}+36 C_{15} \\
& \tilde{Z}_{9}=C_{10}-2 C_{12}+C_{13} .
\end{aligned}
$$

Note that the $\mathrm{SO}(9)$ identity (A.6) is automatically included in these vanishing relations. 


\section{References}

[1] E. Cremmer, B. Julia, and J. Scherk, "Supergravity in 11 dimensions", Phys. Lett. 76B (1978) 409.

[2] K. Peeters, P. Vanhove, and A. Westerberg, "Supersymmetric higher-derivative actions in ten and eleven dimensions, the associated superalgebras and their formulation in superspace", Class. Quant. Grav. 18 (2001) 843-889, hep-th/0010167.

[3] K. Peeters, P. Vanhove, and A. Westerberg, "Towards complete string effective actions beyond leading order", Found. Phys. 52 (2004) 630-635, hep-th/0312211

[4] M. Cederwall, U. Gran, B. E. W. Nilsson, and D. Tsimpis, "Supersymmetric corrections to eleven-dimensional supergravity", JHEP 05 (2005) 052, hep-th/0409107.

[5] M. B. Green, M. Gutperle, and P. Vanhove, "One loop in eleven dimensions", Phys. Lett. B409 (1997) 177-184, hep-th/9706175.

[6] M. B. Green, H. h. Kwon, and P. Vanhove, "Two loops in eleven dimensions", Phys. Rev. D61 (2000) 104010, hep-th/9910055.

[7] E. Cremmer and S. Ferrara, "Formulation of 11-dimensional supergravity in superspace", Phys. Lett. 91B (1980) 61-66.

[8] B. de Wit, K. Peeters, and J. Plefka, "Superspace geometry for supermembrane backgrounds", Nucl. Phys. B532 (1998) 99, hep-th/9803209

[9] M. B. Green, M. Gutperle, and H. H. Kwon, "Light-cone quantum mechanics of the eleven-dimensional superparticle", JHEP 08 (1999) 012, hep-th/9907155

[10] D. J. Gross and J. H. Sloan, "The quartic effective action for the heterotic string", Nucl. Phys. B291 (1987) 41.

[11] A. Dasgupta, H. Nicolai, and J. Plefka, "Vertex operators for the supermembrane", JHEP 05 (2000) 007, hep-th/0003280.

[12] J. Plefka, "Vertex operators for the supermembrane and background field matrix theory", Int. J. Mod. Phys. A16 (2001) 660-668, hep-th/0009193.

[13] L. Anguelova, P. A. Grassi, and P. Vanhove, "Covariant one-loop amplitudes in D=11", Nucl. Phys. B702 (2004) 269-306, hep-th/0408171.

[14] N. Berkovits, "Covariant quantization of the supermembrane", JHEP 09 (2002) 051, hep-th/0201151.

[15] S. Deser and D. Seminara, "Counterterms/M-theory corrections to $D=11$ supergravity", Phys. Rev. Lett. 82 (1999) 2435-2438, hep-th/9812136

[16] S. Deser and D. Seminara, "Tree amplitudes and two-loop counterterms in $D=11$ supergravity", Phys. Rev. D62 (2000) 084010, hep-th/0002241.

[17] S. Deser and D. Seminara, "Graviton-form invariants in $D=11$ supergravity", Phys. Rev. D72 (2005) 027701, hep-th/0506073

[18] T. Banks, W. Fischler, S. H. Shenker, and L. Susskind, "M theory as a matrix model: a conjecture", Phys. Rev. D55 (1997) 5112-5128, hep-th/9610043.

[19] R. Helling, J. Plefka, M. Serone, and A. Waldron, "Three graviton scattering in M-theory", Nucl. Phys. B559 (1999) 184-204, hep-th/9905183.

[20] H. Nicolai and J. Plefka, "A note on the supersymmetric effective action of matrix theory", Phys. Lett. B477 (2000) 309-312, hep-th/0001106. 
[21] B. de Wit, J. Hoppe, and H. Nicolai, "On the quantum mechanics of supermembranes", Nucl. Phys. B305 (1988) 545.

[22] A. Dasgupta, H. Nicolai, and J. Plefka, "An introduction to the quantum supermembrane", Grav. Cosmol. 8 (2002) 1, hep-th/0201182

[23] B. Pioline, H. Nicolai, J. Plefka, and A. Waldron, " $R^{4}$ couplings, the fundamental membrane and exceptional theta correspondences", JHEP 03 (2001) 036, hep-th/0102123.

[24] D. Kazhdan, B. Pioline, and A. Waldron, "Minimal representations, spherical vectors, and exceptional theta series. I", Commun. Math. Phys. 226 (2002) 1-40, hep-th/0107222.

[25] B. Pioline and A. Waldron, "The automorphic membrane", JHEP 06 (2004) 009, hep-th/0404018.

[26] F. Sugino and P. Vanhove, "U-duality from matrix membrane partition function", Phys. Lett. B522 (2001) 145-154, hep-th/0107145

[27] V. Bengtsson, M. Cederwall, H. Larsson, and B. E. W. Nilsson, "U-duality covariant membranes", JHEP 02 (2005) 020, hep-th/0406223

[28] M. B. Green, K. Peeters, and C. Stahn, "Superfield integrals in high dimensions", hep-th/0506161.

[29] S. A. Fulling, R. C. King, B. G. Wybourne, and C. J. Cummins, "Normal forms for tensor polynomials. 1: The Riemann tensor", Class. Quant. Grav. 9 (1992) 1151.

[30] K. Peeters and A. Westerberg, "The Ramond-Ramond sector of string theory beyond leading order", Class. Quant. Grav. 21 (2004) 1643-1666, hep-th/0307298.

[31] K. Peeters, P. Vanhove, and A. Westerberg, "Chiral splitting and world-sheet gravitinos in higher-derivative string amplitudes", Class. Quant. Grav. 19 (2001) 2699-2716, hep-th/0112157.

[32] T. Damour and H. Nicolai, "Higher order M-theory corrections and the Kac-Moody algebra $E_{10}$, hep-th/0504153

[33] V. A. Belinsky, I. M. Khalatnikov, and E. M. Lifshitz, "Oscillatory approach to a singular point in the relativistic cosmology", Adv. Phys. 19 (1970) 525-573.

[34] V. A. Belinsky, I. M. Khalatnikov, and E. M. Lifshitz, "A general solution of the Einstein equations with a time singularity", Adv. Phys. 31 (1982) 639-667.

[35] T. Damour, M. Henneaux, and H. Nicolai, "Cosmological billiards", Class. Quant. Grav. 20 (2003) R145-R200, hep-th/0212256. 